\title{
Molecular analysis of $M V K$ mutations and enzymatic activity in hyper-IgD and periodic fever syndrome
}

\author{
Laurence Cuisset ${ }^{1}$, Joost PH Drenth ${ }^{2}$, Anna Simon ${ }^{3}$, Marie Francoise Vincent ${ }^{4}$, \\ Saskia van der Velde Visser ${ }^{5}$, Jos WM van der Meer ${ }^{3}$, Gilles Grateau ${ }^{6}$, Marc Delpech ${ }^{* 1}$ and \\ and the contributing members of the International Hyper-IgD Study Group
}

${ }^{1}$ Génétique et physiopathologie des maladies inflammatoires héréditaires, Institut Cochin de Génétique Moléculaire and Hôpital Cochin, Assistance Publique-Hôpitaux de Paris, Paris, France; ${ }^{2}$ Department of Medicine, Division of Gastroenterology, University Medical Center St. Radboud, Nijmegen, The Netherlands; ${ }^{3}$ Division of General Internal Medicine University Medical Center St. Radboud, Nijmegen, The Netherlands; ${ }^{4}$ Laboratoire des Maladies Métaboliques, Brussels, Belgium; ${ }^{5}$ Department of Human Genetics, University Medical Center St. Radboud, Nijmegen, The Netherlands; ${ }^{6}$ Service de Médecine Interne, L'Hôtel-Dieu (AP-HP), Paris, France

Hyperimmunoglobulinaemia D and periodic fever syndrome (HIDS) is an autosomal recessive inflammatory disorder characterised by recurrent episode of fever associated with lymphadenopathy, abdominal distress, joint involvement and skin lesions. We recently demonstrated that mutations in the mevalonate kinase gene $(M V K)$ are associated with HIDS. Direct DNA sequencing was done to screen the entire coding region of $M V K$ in 25 unrelated patients with HIDS. Mutations were detected in the coding region of the gene including 11 missense mutations, one deletion, the absence of expression of one allele, as well as three novel polymorphisms. Seven of these mutations are novel. The large majority of the patients were compound heterozygotes for two mutations. Of these, $V 377 I(G \rightarrow A)$ is the most common mutation occurring in 20 unrelated patients and was found to be associated with I268T in six patients. Mutations were associated with a decrease of mevalonate kinase (MK) (ATP:mevalonate 5-phosphotransferase, EC 2.7.1.36) enzymatic activity but not as profound as in mevalonic aciduria, a syndrome also caused by a deficient activity of MK. In HIDS the mutations are located all along the protein which is different from mevalonic aciduria where MK mutations are mainly clustered to a same region of the protein. On the basis of this study, we propose that the diagnostic screen of MVK in HIDS should be first directed on V377I and I268T mutations. Three patients are also described to illustrate the genotypic and phenotypic overlap with mevalonic aciduria. European Journal of Human Genetics (2001) 9, 260-266.

Keywords: Hyper IgD syndrome; periodic fever; mevalonate kinase

\section{Introduction}

The hyperimmunoglobulinaemia $\mathrm{D}$ and periodic fever syndrome (HIDS (MIM 260920), http://www.hids.net) is an autosomal recessive disorder with recurrent febrile attacks as
*Correspondence: Marc Delpech, INSERM EMI-05, Génétique et physiopathologie des maladies inflammatoires héréditaires, Institut Cochin de Génétique Moléculaire, 24 rue du faubourg Saint-Jacques, 75014 Paris, France. Tel: +331584116 14; Fax: +33144411522 or +33158 4115 80; E-mail: delpech@icgm.cochin.inserm.fr Received 9 August 2000; revised 17 November 2000; accepted 20 November 2000 its main feature. ${ }^{1,2}$ During these attacks patients also suffer from a variable presence of lymphadenopathy, arthralgias, splenomegaly, skin lesions and headache. Symptoms start in early childhood and may persist during life. So far, 13 HIDS families with two or more affected siblings are known to us. The elevated serum immunoglobulinaemia D (IgD; to values beyond $100 \mathrm{IU} / \mathrm{ml}$ ) serves as convenient biochemical marker. ${ }^{3}$ The clinical presentation is rather homogeneous although severity and intensity of the febrile crises may differ among patients. A recent genome wide search linked HIDS to the long arm of chromosome $12(12 q 24)^{4}$ and the linked interval contains the gene encoding for mevalonate 
kinase $(M V K) . M K$, a homodimeric enzyme present in the peroxisomes of every mammalian cell, follows 3-hydroxy-3methylglutaryl-CoA reductase in the cholesterol synthesis and converts mevalonate into 5-phosphomevalonate. A near complete deficiency of this enzyme causes mevalonic aciduria (MIM 251170). This rare autosomal recessive disorder is characterised by developmental delay, failure to thrive, hypotonia, ataxia, myopathy, and cataracts. Most notably, many patients suffer from recurrent febrile attacks with vomiting, diarrhoea, leukocytosis, elevated erythrocyte sedimentation rate, arthralgia, and morbilliformic rash. ${ }^{5,6}$ These phenotypical similarities with HIDS lead us to consider mevalonate kinase as a candidate gene for HIDS. Effective linkage and the serendipitous finding by others of minor elevated urinary excretions of mevalonic acid in a HIDS patient, led to the identification of $M V K$ as the causative HIDS gene. ${ }^{4,7}$ Preliminary molecular analysis revealed four different missense mutations (V377I, I268T, H20P and P167L), the absence of expression of one allele and a 92-bp deletion in the $M V K$ gene. These mutations result in a functional defect of MK and preliminary studies indicated a residual activity of $5-15 \%$, values higher than those observed in mevalonic aciduria. At present it is unclear whether HIDS and mevalonic aciduria are extremes of a continuous spectrum or that they are truly two different entities. For example, two mutations (I268T and H20P) are common in HIDS and mevalonic aciduria patients, but have been reported heteroallelic with different mutations in both. ${ }^{8,9}$ Therefore, the different nature of HIDS and mevalonic aciduria may be caused by a differential mutational spectrum, but information to this end is lacking.

Therefore, we have undertaken studies in order to gain more insight in the genotypes in a large cohort of HIDS patients. We expected to shed light on the molecular pathology of HIDS and to aid the development of an effective molecular testing strategy for diagnosis and carrier testing.

\section{Subjects and methods \\ Patients}

Patients for this study were selected on the basis of previous inclusion in the Nijmegen HIDS registry ${ }^{1}$ and the availability of Epstein-Barr immortalised cell lines. The Nijmegen HIDS registry was set up in 1992 and hosts clinical and laboratory data on these patients. Twenty-five unrelated patients agreed to participate in the present study and in eight cases, father and mother were included. The numbering of the patients reflects the entry number in the Nijmegen HIDS registry. A thorough clinical examination was performed on all subjects and HIDS was diagnosed in concordance to set criteria. ${ }^{1}$ Biochemical proof of affected status was obtained by measurement of the IgD content in serum. The hereditary pattern in all included families was compatible with an autosomal recessive hereditary trait. ${ }^{1}$ The study was carried out after informed consent from all individuals and formal approval was obtained by the Medical Ethical Comittee (CWOM) of the University Medical Center St. Radboud, Nijmegen, The Netherlands.

\section{Databases}

Online Mendelian Inheritance in Man (OMIM) is http:// www.ncbi.nlm.nih.gov (for HIDS (MIM 260920) and mevalonic aciduria (MIM 251170)). Blast searches of the high throughput genomic sequences (htgs) database of the National Center for Biotechnology Information (NCBI; http://www.ncbi.nlm.nih.gov) were performed using the cDNA sequence of MVK (GeneBank M88468).

\section{Screening of $M V K$ mutations}

RNA was extracted from Epstein-Barr immortalised cell lines according to established protocols. Total RNA was prepared using the RNA B reagent (Bioprobe) according to the instructions of the manufacturer. Four micrograms of RNA were used to synthetise cDNA with Superscript reverse transcriptase II (Gibco BRL), followed by PCR using Platinium Taq DNA polymerase (Gibco BRL) in a GeneAmp PCR system 9600 (Perkin Elmer). We determined the nucleotide sequences of the amplified fragments by standard semiautomated methods on an ABI PRISM 377 (Perkin Elmer).The primers used in this study have already been described. ${ }^{4}$ The complete coding region of $M V K$ was amplified from cDNA in two overlapping fragments using primers 29/788 and 591/ 1381. Their sequences were determined using primers 29, $381,535,921,1000$ and 1381. When the homozygous status of a mutation in a proband could not be controlled by the analysis of both parents, an additional PCR was performed on cDNA using primers 381/1000 followed by sequence analysis to eliminate the presence of a short deletion spanning primers 788 and 591.

\section{MK enzyme analysis}

Mevalonate kinase enzyme activity in extracts of cultured lymphocytes were performed by a radiometric assay. DL[Mevalonic-2- ${ }^{14} \mathrm{C}$ ], DBED salt was purchased from NEN Life Science Products, Inc. (Boston, MA, USA). Non-isotopic DLmevalonolactone, from Sigma Chemical (St Louis, MO, USA), was converted before use to the potassium salt as described. ${ }^{10}$ Preparation of the lymphoblasts lysates was carried out as described earlier. ${ }^{11}$ Mevalonate kinase assay in lymphoblast lysates, including separation of substrate and products by thin layer chromatography, was performed according to Gibson et al. ${ }^{10}$

\section{Results}

\section{Clinical data}

The study group consisted of 25 patients: 22 Dutch, one British, one Czech, one Spanish. The mean age was $30.3 \pm 16.4$ years and there was an even male-female ratio (13 females and 12 males). The clinical features of the first 50 
patients in the Nijmegen HIDS registry have been described in detail elsewhere. ${ }^{1}$ Briefly, all patients had recurrent attacks of fever $\left(38.5^{\circ} \mathrm{C}\right)$ with an acute phase response with elevated erythrocyte sedimentation rate and a brisk leukocytosis. All had a constantly elevated serum $\operatorname{IgD}(>100 \mathrm{U} / \mathrm{ml})$ measured on two occasions at least 1 month apart, and one or more of the following symptoms during attacks; lymphadenopathy; abdominal distress (vomiting, diarrhoea, pain); skin manifestations (erythematous macules and papules); arthralgias/ arthritis; and splenomegaly. Although affected siblings were available for the study we elected to study unrelated patients in order to obtain a representative survey of mutational spectrum and enzyme activities of mevalonate kinase in HIDS.

\section{Genomic structure of the MVK gene}

The genomic organization of the MVK gene is shown in Figure 1 and was determined to help in the understanding of the deletion found in HIDS patients. We deduced it by comparison between a human genomic clone from the working draft sequencing database (accession number AC007623) and the human cDNA (accession number M88468). The gene contains 10 coding exons and one non coding exon spanning over $21 \mathrm{~kb}$. The size of the exons varies from 62 to $837 \mathrm{bp}$ and the size of the introns from 378 to $4470 \mathrm{bp}$. The first and last base belonging to each exon is indicated in Figure 1 and refers to the numbering of the human cDNA. The last exon contains $152 \mathrm{bp}$ of the open reading frame (ORF) and $685 \mathrm{bp}$ of the $3^{\prime}$ UTR.

\section{Mutation analysis}

Of the 25 unrelated patients investigated for sequence variations in the coding region of the $M V K$ gene, we were able to identify a total of 13 mutations and three new polymorphisms. Seven mutations are novel and have never been described before. Mutations were detected in all exons except in exon 3 and 7 (Table 1). Most of the mutations are missense mutations; nonsense mutations were not found. The position of the affected nucleotide refers to the human MVK cDNA (accession number M88468). Where available both parents were studied (patients: 17, 21, 26, 31, 35, 47, 100, 116).

\section{Missense mutations}

We found seven new mutations: R215Q, L39P, G202R, G326R, G309S, S150L and H20N. Each of them were detected on only one allele in seven unrelated patients indicating that they are not very frequent in HIDS.

Among the previously reported $M V K$ mutations we detected the four known HIDS missense mutations V377I, I268T, H20P and P167L, and surprisingly one patient carried the A334T allele which previously had only been described in mevalonic aciduria.

V377I, which is located at the C-terminal end of the protein, was found in 20 of 25 patients and therefore constitutes the most frequent mutation in HIDS. I268T occurred in seven unrelated patients and is the second most frequent mutation in HIDS. V377I and I268T were found heteroallelic in six patients. Interestingly, one patient carrying I268T was found homozygous for that mutation. The status of the parents could not be determined as they were both dead at the start of the study. Patient 116 was found homozygous for P167L.

\section{Absence of expression of one allele}

Analysis of the parents of this last patient (patient 116) showed that the mother was a heterozygote for P167L and that the father displayed a normal allele, suggesting that the second mutation in the proband led to the absence of expression of the second allele. In the group of the 20 patients carrying V377I $([1220 \mathrm{G} \rightarrow \mathrm{A}])$, six were found to be homozygous upon examination on cDNA. For only three of them, both parents were available (patients 17, 26 and 35) and tested. In these three families, one parent was heterozygous G/A at position 1220 whereas the other parent was found to be homozygous for the normal $G$ at the same position. This observation has been reported in our first study ${ }^{4}$ and reveals that in these three families the patient may carry either an unknown mutation or a deletion of the whole genomic sequence on the second allele that leads to the absence of expression of mRNA, or a fresh mutation at a hyper mutable point. Unfortunately, patients 15,72 and 89 could not be investigated further because samples from their parents and genomic DNA from these three patients were not

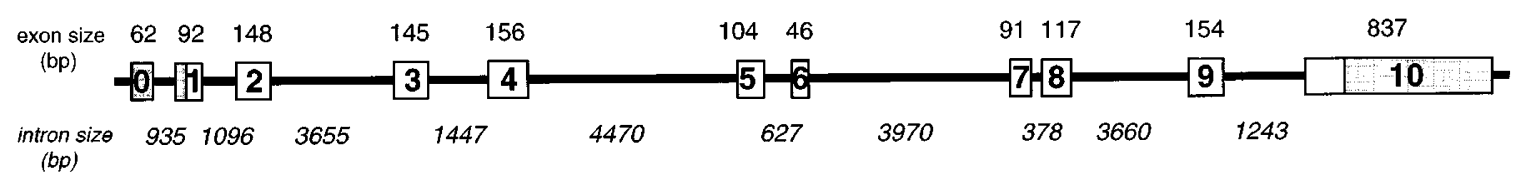

\begin{tabular}{|lccc|}
\hline \multicolumn{4}{|c|}{ exons (nucleotide position) } \\
$0: 11-77$ & $3: 318-462$ & $6: 723-768$ & $9: 978-1130$ \\
$1: 78-169$ & $4: 463-618$ & $7: 769-859$ & $10: 1131-1282$ \\
$2: 170-317$ & $5: 619-722$ & $8: 860-977$ & \\
\hline
\end{tabular}

coding sequence non coding sequence

Figure 1 Genomic organization of the MVK gene. This structure has been deduced by comparison between a genomic clone (AC007623) and the human CDNA (M88468). In the large square are indicated the first and last nucleotide position belonging to each exon and refers to the numbering of the human cDNA. 
Table 1 Mutations in the MVK cDNA

\begin{tabular}{|c|c|c|c|c|}
\hline $\begin{array}{l}\text { Patient } \\
\text { no }\end{array}$ & Exon & $\begin{array}{l}\text { Nucleotide } \\
\text { change }\end{array}$ & $\begin{array}{l}\text { Amino acid } \\
\text { change }^{a}\end{array}$ & $\begin{array}{l}\text { MK enzyme } \\
\text { activity }^{b}\end{array}$ \\
\hline 1 & $\begin{array}{r}10 \\
?\end{array}$ & $\begin{array}{c}1220 \mathrm{G} \rightarrow \mathrm{A} \\
?\end{array}$ & $\begin{array}{c}\text { V377I } \\
?\end{array}$ & 8.9 \\
\hline 4 & $\begin{array}{r}8 \\
10\end{array}$ & $\begin{array}{l}894 \mathrm{~T} \rightarrow \mathrm{C} \\
1220 \mathrm{G} \rightarrow \mathrm{A}\end{array}$ & $\begin{array}{l}\text { I268T } \\
\text { V377I }\end{array}$ & 5 \\
\hline 10 & $\begin{array}{r}9 \\
10\end{array}$ & $\begin{array}{l}1016 \mathrm{G} \rightarrow \mathrm{A} \\
1220 \mathrm{G} \rightarrow \mathrm{A}\end{array}$ & $\begin{array}{l}\text { G309S } \\
\text { V377I }\end{array}$ & 3.6 \\
\hline 11 & $\begin{array}{r}1 \\
10\end{array}$ & $\begin{array}{l}150 \mathrm{~A} \rightarrow \mathrm{C} \\
1220 \mathrm{G} \rightarrow \mathrm{A}\end{array}$ & $\begin{array}{l}\text { H2OP } \\
\text { V377I }\end{array}$ & 8.5 \\
\hline 12 & $\begin{array}{r}2 \\
10\end{array}$ & $\begin{array}{l}207 \mathrm{~T} \rightarrow \mathrm{C} \\
1220 \mathrm{G} \rightarrow \mathrm{A}\end{array}$ & $\begin{array}{l}\text { L39P } \\
\text { V377I }\end{array}$ & 2.1 \\
\hline 13 & $\begin{array}{r}8 \\
10\end{array}$ & $\begin{array}{l}894 \mathrm{~T} \rightarrow \mathrm{C} \\
1220 \mathrm{G} \rightarrow \mathrm{A}\end{array}$ & $\begin{array}{l}1268 \mathrm{~T} \\
\text { V377I }\end{array}$ & 7.2 \\
\hline 15 & $\begin{array}{r}10 \\
?\end{array}$ & $\begin{array}{c}1200 \mathrm{G} \rightarrow \mathrm{A} \\
?\end{array}$ & $\begin{array}{c}\text { V377I homo } \\
?\end{array}$ & 4.5 \\
\hline 17 & $\begin{array}{c}10 \\
\text { ND }\end{array}$ & $\begin{array}{l}1220 G \rightarrow A \\
N D\end{array}$ & $\begin{array}{l}\text { V377I homo } \\
\text { Abs RNA }\end{array}$ & 28 \\
\hline 18 & $\begin{array}{r}8 \\
10\end{array}$ & $\begin{array}{l}894 \mathrm{~T} \rightarrow \mathrm{C} \\
1220 \mathrm{G} \rightarrow \mathrm{A}\end{array}$ & $\begin{array}{l}\text { I268T } \\
\text { V377I }\end{array}$ & 4 \\
\hline 21 & $\begin{array}{r}1 \\
10\end{array}$ & $\begin{array}{l}150 \mathrm{~A} \rightarrow \mathrm{C} \\
1220 \mathrm{G} \rightarrow \mathrm{A}\end{array}$ & $\begin{array}{l}\mathrm{H} 20 \mathrm{P} \\
\text { V3771 }\end{array}$ & 11.4 \\
\hline 26 & $\begin{array}{l}10 \\
\text { ND }\end{array}$ & $\begin{array}{l}1220 G \rightarrow A \\
N D\end{array}$ & $\begin{array}{l}\text { V377I homo } \\
\text { Abs RNA }\end{array}$ & 11.3 \\
\hline 31 & $\begin{array}{r}8 \\
10\end{array}$ & $\begin{array}{l}894 \mathrm{~T} \rightarrow \mathrm{C} \\
1220 \mathrm{G} \rightarrow \mathrm{A}\end{array}$ & $\begin{array}{l}\text { I268T } \\
\text { V377I }\end{array}$ & 15.3 \\
\hline 35 & $\begin{array}{r}10 \\
\text { ND }\end{array}$ & $\begin{array}{l}1200 G \rightarrow A \\
N D\end{array}$ & $\begin{array}{l}\text { V377I homo } \\
\text { Abs RNA }\end{array}$ & ND \\
\hline 47 & $\begin{array}{l}1 \\
6\end{array}$ & $\begin{array}{l}149 C \rightarrow A \\
735 G \rightarrow A\end{array}$ & $\begin{array}{l}\mathrm{H} 20 \mathrm{~N} \\
\mathrm{R} 215 \mathrm{Q}\end{array}$ & 3.5 \\
\hline 52 & $\begin{array}{l}4 \\
5\end{array}$ & $\begin{array}{l}591 C \rightarrow T \\
695 G \rightarrow A\end{array}$ & $\begin{array}{l}\text { P167L } \\
\text { G202R }\end{array}$ & 1.8 \\
\hline 55 & $\begin{array}{r}8 \\
10\end{array}$ & $\begin{array}{l}894 \mathrm{~T} \rightarrow \mathrm{C} \\
1220 \mathrm{G} \rightarrow \mathrm{A}\end{array}$ & $\begin{array}{l}\text { I268T } \\
\text { V377I }\end{array}$ & 10.7 \\
\hline 56 & $\begin{array}{r}8 \\
10\end{array}$ & $\begin{array}{l}894 \mathrm{~T} \rightarrow \mathrm{C} \\
1220 \mathrm{G} \rightarrow \mathrm{A}\end{array}$ & $\begin{array}{l}1268 T \\
\text { V377I }\end{array}$ & 7.7 \\
\hline 61 & $\begin{array}{r}4 \\
10\end{array}$ & $\begin{array}{l}540 \mathrm{C} \rightarrow \mathrm{T} \\
1220 \mathrm{G} \rightarrow \mathrm{A}\end{array}$ & $\begin{array}{l}\text { S150L } \\
\text { V377I }\end{array}$ & 17.3 \\
\hline 66 & $\begin{array}{r}9 \\
10\end{array}$ & $\begin{array}{l}1067 G \rightarrow A \\
1220 G \rightarrow A\end{array}$ & $\begin{array}{l}\text { G326R } \\
\text { V377I }\end{array}$ & 16.4 \\
\hline 71 & $\begin{array}{l}9 \\
?\end{array}$ & $\begin{array}{c}1091 \mathrm{G} \rightarrow \mathrm{A} \\
?\end{array}$ & $\begin{array}{r}\mathrm{A} 334 \mathrm{~T} \\
?\end{array}$ & 0 \\
\hline 72 & $\begin{array}{r}10 \\
?\end{array}$ & $\begin{array}{c}1220 \mathrm{G} \rightarrow \mathrm{A} \\
?\end{array}$ & $\begin{array}{c}\text { V377I homo } \\
?\end{array}$ & 8 \\
\hline 89 & $\begin{array}{r}10 \\
?\end{array}$ & $\begin{array}{c}1220 \mathrm{G} \rightarrow \mathrm{A} \\
?\end{array}$ & $\begin{array}{c}\text { V377I homo } \\
?\end{array}$ & 5.7 \\
\hline 100 & $\begin{array}{r}4 \\
10\end{array}$ & $\begin{array}{l}465-619 \text { del } \\
1220 G \rightarrow A\end{array}$ & $\begin{array}{l}\text { del exon } 4 \\
\text { V3771 }\end{array}$ & 8.3 \\
\hline 103 & $\begin{array}{l}8 \\
?\end{array}$ & $\begin{array}{c}894 \mathrm{~T} \rightarrow \mathrm{C} \\
?\end{array}$ & $\begin{array}{c}\text { I268T homo } \\
?\end{array}$ & 7.3 \\
\hline 116 & $\begin{array}{c}4 \\
\text { ND }\end{array}$ & $\begin{array}{l}591 C \rightarrow T \\
N D\end{array}$ & $\begin{array}{l}\text { P167L homo } \\
\text { Abs RNA }\end{array}$ & 9.7 \\
\hline
\end{tabular}

del=deletion, homo=homozygote, $\mathrm{ND}=$ not determined and abs

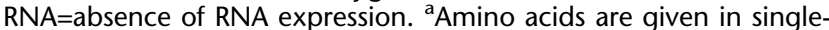
letter code. ${ }^{\mathrm{b}}$ Enzyme activities are derived from experiments using cultured lymphoblasts and are expressed as percentage of control.

available. Additional RT-PCR using other sets of primers spanning primers 788 and 591 followed by sequencing analysis did not reveal any other alterations. However it cannot be excluded that these patients carry a mutation spanning the $5^{\prime}$ or $3^{\prime}$ end of the cDNA that would prevent the primer annealing.

\section{Deletions}

In patient 100 we found a deletion spanning from nucleotide 465 to 619 . Comparison with the deduced genomic organization reveals that this mutation corresponds to the complete deletion of exon 4 suggesting that this patient would carry a mutation affecting the splicing of the transcript. The same deletion was found in her healthy father, whereas the second mutation of the patient (V377I) was transmitted by her healthy mother. The determination of the genomic structure allowed us to conclude that the $92 \mathrm{bp}$ deletion we reported for family 13 in our previous study ${ }^{4}$ corresponds to a complete deletion of exon 1 suggesting that this patient would also carry a mutation affecting the splicing of one transcript.

\section{Polymorphisms}

Three novel polymorphisms were identified. A $601 \mathrm{C} \rightarrow \mathrm{T}$ transition within exon 4 resulting in a silent change $(170 \mathrm{D} \rightarrow \mathrm{D})$ was found in $5 \%$ of the individuals analysed. Another polymorphism, $246 \mathrm{G} \rightarrow \mathrm{A}$ with a resulting $52 \mathrm{~S} \rightarrow \mathrm{N}$, was detected in $10 \%$ of individuals. Although this modification changes the amino acid sequence, we expect it to be silent because it was found in one healthy mother and one independent healthy father (both carrying another heterozygous mutation). In addition, it did not segregate with HIDS as it was not transmitted to their respective affected children. The functional significance of this polymorphism is not known. In addition to these missenses, a 1336insG was found. This polymorphism was located $50 \mathrm{bp}$ after the stop codon and therefore did not affect the coding sequence. It was found in $5 \%$ of individuals including a healthy father (carrying another heterozygous mutation) who did not transmit this polymorphism to his affected children. None of these rare polymorphisms were specifically associated with a mutated allele and therefore did not give any informations about a potential founder effect in the disease.

\section{MK activity}

Analysis of the MK enzyme activities in cultured lymphoblasts were greatly affected in HIDS patients. When we excluded patient 71, who had a nearly absent MK activity, the average activity was decreased to $9.2 \%$ (SD 6\%) of that of healthy controls (Table 1). There was a great variation among HIDS patients and enzyme activities varied from $1.8 \%$ to as much as $28 \%$.

\section{Position of the mutations}

To analyse the distribution of mutations in the MK protein sequence, we combined our data with results from previous reports $^{4,7-9,12,13}$ and plotted mutations in HIDS and mevalonic aciduria against amino acids residues (Figure 2). This shows that the HIDS mutations are evenly distributed along the coding region of $M V K$, which contrasts to that of mevalonic aciduria which essentially clusters between amino acids 243 and 334. Except for H2OP 


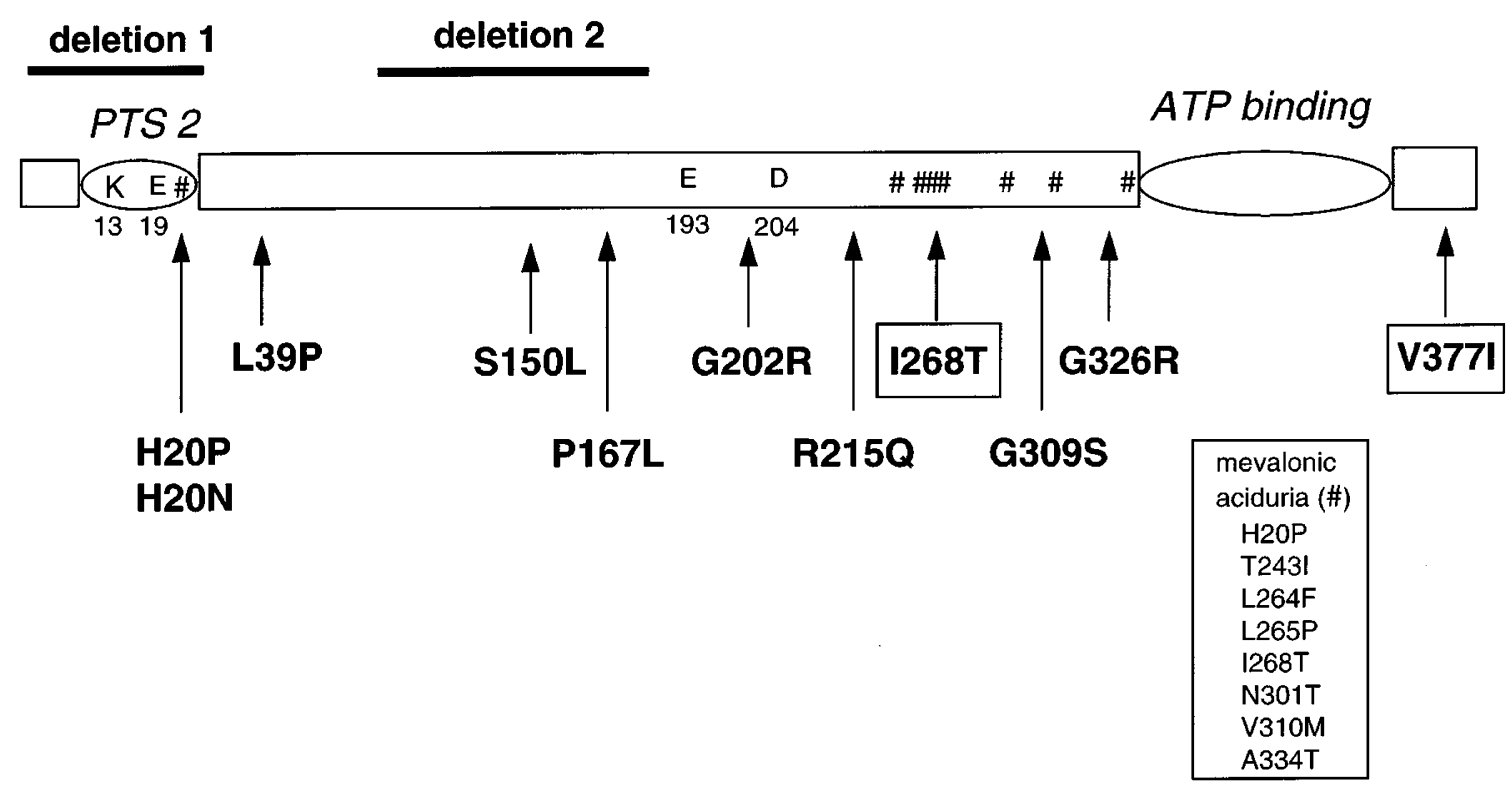

Figure 2 Summary of mutations identified in HIDS and mevalonic aciduria patients. ${ }^{4,7-9,12,13}$ The bar depicts the MK protein. PTS2 homology domain and putative ATP binding domain are indicated. K13, E19, E193 and D204 are known important functional amino. $^{14,15}$ Deletion 1 refers to our previous report ${ }^{4}$ and deletion 2 refers to this study. HIDS mutations are indicated in bold characters and mevalonic aciduria mutations (\#) are summarised in the large square.

and I268T, none of the mutations are common between HIDS and mevalonic aciduria. MK contains a peroxisomes targeting sequence 2 (PTS2) domain in the $\mathrm{N}$ terminal part and an ATP binding site in the $\mathrm{C}$ terminal part. Four amino acids (K13, E19, E193 and D204) have been shown to play a crucial role in the enzymatic activity of the protein ${ }^{14,15}$ and some of the HIDS mutations are very close to these important amino acids.

\section{Genotype-phenotype correlation}

To evaluate the functional significance of the mutations identified we undertook a careful evaluation of the phenotypes and compared them with the different types of the mutations observed. Patients were evaluated for age of onset of disease, frequency and length of attacks and symptoms accompanying the attacks. Laboratory data with emphasis on immunoglobulins and parameters of the acute phase response were monitored. We compared the phenotype of those who were compound heterozygotes for V377I to the five patients who did not carry this mutation. The frequency or severity of febrile attacks did not differ between V377I positive and negative patients, nor was there a difference in symptomatology during attacks.

One patient, a 39-year-old woman (71) carried an A344T mutation on one allele. This mutation is thought to be associated with mevalonic aciduria. ${ }^{13}$ She had typical febrile attacks from birth onwards but also developed a tapetoretinal blindness and optical nerve atrophy at the age of 2 . After an episode of measles with encephalitis she developed a clinical picture of severe mental retardation, cerebellar ataxia, athethosis, and microcephalia with epilepsy. She continued to have febrile attacks. Immunoglobulins are elevated: IgD (465 IU/ml) and $\operatorname{IgA}(4 \mathrm{~g} / \mathrm{l})$. The MK enzyme activity was $<1 \%$ and the excretion of mevalonic acid in urine was strongly elevated $7200 \mathrm{mmol} / \mathrm{mol}$ creatinine. On the basis of the massive urinary mevalonic acid excretion and the complete absence of enzymatic activity she must now be classified as having mevalonic aciduria instead of HIDS. It is likely that the severe neurological symptoms fit with mevalonic aciduria and cannot be attributed as a sequel to measles encephalitis.

Another patient (15) had a phenotype similar to that of patient 71, with mental retardation, tapetoretinal blindness, cataracts, and ataxia. In contrast, he had the V377I mutation, the MK activity was $4.5 \%$ of controls and during remission there was no excretion of mevalonic acid. This labelled him as HIDS. Patient 47, a 20-year-old man who was a compound heterozygote for $\mathrm{H} 20 \mathrm{~N}$ and $\mathrm{R} 215 \mathrm{Q}$ had a very severe phenotype. He had repetitive febrile attacks with deforming arthritis. Patient 52, who was compound heterozygote for P167L and G202R, died of staphylococcal pneumonia at the age of 30 but she did not have any attacks in the 18 months preceeding her death. Patient 103, a 40-year-old male, was a homozygote for I268T. Neurological examination was completely normal and he had no dysmorphic features. He had a very mild disease with only $3-4$ febrile attacks per year. The sequence variation has been reported before in mevalonic aciduria. $^{8}$ The last patient (116) was a 10-year-old girl who was a compound heterozygote for the P167L mutation and had the absence of expression of the second allele. She had 
regular febrile attacks but growth and development was unremarkable.

\section{Discussion}

Our study presents detailed clinical and mutation analysis data on the largest cohort of patients with HIDS. Although affected siblings were available, we elected to study a total of 25 unrelated patients to obtain a representative survey of mutations in the $M V K$ gene and enzyme activity. We identified 13 different mutations, seven of which were novel, comprising 11 missense mutations, one deletion and the absence of expression of one allele. Three novel polymorphisms were found. Missense mutations of $M V K$ were detected at the heterozygous stage on both alleles of 14 patients and on one allele of two patients ( 1 and 71$)$. In these two last patients the first heterozygous mutation was localised in the 3 ' part of the cDNA and the second mutation could not be determined. The disease manifestation in these two patients may be explained by a short deletion overlapping the forward primer of the $5^{\prime}$ part of the cDNA or by a mutation in intronic sequences as these were not addressed in this study. We consider patient 1 as typical HIDS because she possessed the most frequent $M V K$ mutation (V377I) and displayed the classical features of HIDS, being the first HIDS patient diagnosed. ${ }^{16}$ This further study allows us to draw a more complete picture of the biochemical and mutational differences between HIDS and mevalonic aciduria. In our HIDS cohort the MK enzyme activities vary widely and, as we previously observed, ${ }^{4}$ the lower values may overlap with those observed in mevalonic aciduria. ${ }^{6}$

However, in mevalonic aciduria, there are very high concentrations of mevalonic acid present constitutively in all bodyfluids, most notably in urine. This is clearly different from HIDS, where only a minor elevated excretion of urinary mevalonic acid can be observed with attacks. In our cohort we detected a patient with grossly elevated urinary mevalonic acid excretion. This patient (71), a 39-year-old woman presented with febrile attacks typical for HIDS but was heterozygous for A334T, an allele that had already been reported in patients with mevalonic aciduria. On the basis of the increased excretion of mevalonic acid in urine we label her now with mevalonic aciduria. Another patient (15) with similar phenotypical features, carried the V377I mutation and lacked mevalonic aciduria. Both patients illustrated the phenotypic overlap between mevalonic aciduria and HIDS. Patient 71 probably represented a mild phenotype of mevalonic aciduria and most notably, she had an unusually long survival. The course of this syndrome is almost invariably fatal and although some patients may reach late adolescence, survival beyond 35 years has not been described before. ${ }^{6}$ Patient 15 was a prototype of a severe classical HIDS patient.

Interestingly, mutations $\mathrm{H} 20 \mathrm{P}$ and $\mathrm{I} 268 \mathrm{~T}$ which are shared by both HIDS and mevalonic aciduria diseases, were detected in our sample. In most HIDS patients we found it in association with V377I which suggests that this mutation is responsible for the HIDS phenotype. This corroborates with data generated by expression of MVK cDNA's in E. coli lysates which only showed a decrease of MK enzyme activity to approximately one-third of controls. ${ }^{7}$ In previously reported mevalonic aciduria cases, H2OP and I268T were found associated with A334T and N301T respectively ${ }^{8,9}$ suggesting that these last two mutations would determine the severity of the phenotype. However, our finding of a HIDS patient who was homozygous for I268T was puzzling given the fact that homozygosity for this mutation was previously found in a patient with very severe mevalonic aciduria. The patient had a nearly absent MK activity and died at the age of 4.5 months. ${ }^{8}$ It is intriguing that our HIDS patient lacked the phenotypical characteristics of mevalonic aciduria such as psychomotor retardation, hypotonia, myopathy and ataxia. He possesed a residual enzyme activity of 7.2\%, far higher than that in mevalonic aciduria. These findings provide the first evidence that the two diseases can share the same $M V K$ mutations. This suggests that not only these specific mutations but also other, as yet undetermined, factors are important for the resultant phenotype.

Our study also clearly demonstrates that the V377I mutation, as a consequence of a $\mathrm{G}$ to A transition, is the most frequent HIDS mutation as it was detected in 20 patients. We also provide evidence that this mutation is not systematically associated with the HIDS phenotype since four patients did not possess the mutant V377I allele. The second most frequent mutation in HIDS is I268T and was found in seven patients. In six of them, patients were compound heterozygotes with V377I. On the basis of these findings we recommend that a diagnostic screen of the $M V K$ gene for HIDS mutations should first be directed on V377I and I268T because they both constitute the most common mutations in HIDS. Since V377I abolishes a BsmAI site ${ }^{4}$ the detection of V377I with restriction enzymes can be done rapidly and this probably represents a very useful tool for molecular diagnosis of HIDS.

Seven patients were found to display a homozygous mutation using cDNA. For four of them, analysis of both parents allowed us to demonstrate that they carried a second unknown mutation that led to the absence of expression of the second allele. We previously reported such observations ${ }^{4}$ and the detection of four additional patients here suggested this was a relatively frequent event in HIDS. The finding of one common mutation (V377I) suggests however that our families are distantly related and suggests that it could be a founder mutation. Indeed most patients described in this report stem from the Netherlands, in concordance with the founder hypothesis. Unfortunately, the rare polymorphisms found in the cDNA could not address this question and therefore this issue must be directed by analysing haplotypes generated by microsatellite markers or by single nucleotide polymorphisms within the gene. 


\section{Acknowledgements}

We thank the patients and parents for their cooperation in pursuing this study. The following members of the International Hyper-IgD Study Group helped to collect DNA from the patients: CMR Weemaes, J Bijlstra, ER Espanol, D Jilek, J Mydlil, S Kynclova, V Kredbova, ER de Graeff Meeder, I Louis. We thank Franck Letourneur and Nicolas Lebrun for sequencing. Joost PH Drenth is a recepient of a grant of the Niels Stensen Foundation. JPH Drenth is an Investigator of the Royal Dutch Academy of Arts and Sciences. Anna Simon is a recipient of a Dutch Organization for Scientific Research fellowship for Clinical Investigators (KWO 920-03-116).

\section{References}

1 Drenth JP, Haagsma CJ, van der Meer JW: Hyperimmunoglobulinemia $\mathrm{D}$ and periodic fever syndrome. The clinical spectrum in a series of 50 patients. International Hyper-IgD Study Group. Medicine (Baltimore) 1994; 73: $133-144$.

2 Drenth JP, van der Meer JW: Periodic fever, entering the era of molecular diagnosis, and they are throwing some light on inflammatory mechanisms. BMJ 2000; 320: 1091-1092.

3 Drenth JP, Klasen IS, van der Meer JW: Recognition of IgD and periodic fever. Ann Intern Med 1996; 125: 518.

4 Drenth JP, Cuisset L, Grateau G et al: Mutations in the gene encoding mevalonate kinase cause hyper-IgD and periodic fever syndrome. International Hyper-IgD Study Group. Nature Genet 1999; 22: $178-181$.

5 Hoffmann G, Gibson KM, Brandt IK, Bader PI, Wappner RS, Sweetman L: Mevalonic aciduria - an inborn error of cholesterol and nonsterol isoprene biosynthesis. New Engl J Med 1986; 314: $1610-1614$

6 Hoffmann GF, Charpentier C, Mayatepek E et al: Clinical and biochemical phenotype in 11 patients with mevalonic aciduria. Pediatrics 1993; 91: 915 -921.

7 Houten SM, Kuis W, Duran M et al: Mutations in MVK, encoding mevalonate kinase, cause hyperimmunoglobulinaemia D and periodic fever syndrome. Nature Genet 1999; 22: 175-177.
8 Houten SM, Romeijn GJ, Koster J et al: Identification and characterization of three novel missense mutations in mevalonate kinase cDNA causing mevalonic aciduria, a disorder of isoprene biosynthesis. Hum Mol Genet 1999; 8: 1523-1528.

9 Hinson DD, Ross RM, Krisans S et al: Identification of a mutation cluster in mevalonate kinase deficiency, including a new mutation in a patient of mennonite ancestry. Am J Hum Genet 1999; 65: $327-335$

10 Gibson KM, Lohr JL, Broock RL et al: Mevalonate kinase in lysates of cultured human fibroblasts and lymphoblasts: kinetic properties, assay conditions, carrier detection and measurement of residual activity in a patient with mevalonic aciduria. Enzyme 1989; 41: $47-55$.

11 Hoffmann GF, Brendel SU, Scharfschwerdt SR, Shin YS, Speidel IM, Gibson KM: Mevalonate kinase assay using DEAE-cellulose column chromatography for first-trimester prenatal diagnosis and complementation analysis in mevalonic aciduria. J Inherit Metab Dis 1992; 15: 738-746.

12 Schafer BL, Bishop RW, Kratunis VJ et al: Molecular cloning of human mevalonate kinase and identification of a missense mutation in the genetic disease mevalonic aciduria. J Biol Chem 1992; 267: $13229-13238$.

13 Hinson DD, Chambliss KL, Hoffmann GF, Krisans S, Keller RK, Gibson KM: Identification of an active site alanine in mevalonate kinase through characterization of a novel mutation in mevalonate kinase deficiency. J Biol Chem 1997; 272: 26756-26760.

14 Potter D, Miziorko HM: Identification of catalytic residues in human mevalonate kinase. J Biol Chem 1997; 272: 2544925454.

15 Potter D, Wojnar JM, Narasimhan C, Miziorko HM: Identification and functional characterization of an active-site lysine in mevalonate kinase. J Biol Chem 1997; 272: 5741-5746.

16 van der Meer JW, Vossen JM, Radl J et al: Hyperimmunoglobulinaemia D and periodic fever: a new syndrome. Lancet 1984; 1 : $1087-1090$. 